\title{
Protocolo libre de opioides en el Hospital Universitario Austral para pacientes sometidos a cirugía de bariátrica
}

Ponte T. ${ }^{1}$, Gamboz F. ${ }^{1}$

1 Hospital Universitario Austral, Pilar, Argentina.

Introducción: El desarrollo de cirugía bariátrica laparoscópica y la integración de un equipo multidisciplinario han hecho que esta se convierta en el tratamiento más eficaz y eficiente contra la obesidad severa y sus comorbilidades.

Desde el punto de vista de la anestesia, el desarrollo de técnicas alternativas libre de opioides para el manejo intraoperatorio ha demostrado tener una menor incidencia de efectos adversos en el post operatorio inmediato.

Objetivos: Primario: describir la anestesia libre de opiáceos.

Secundarios:

- Describir intensidad de dolor postoperatorio en UCPA.

-Describir la cantidad de rescates analgésicos y tiempo transcurrido desde el ingreso hasta el alta.

-Incidencia de náuseas y vómitos.

Materiales y Métodos: Se realizará un estudio descriptivo en pacientes que han recibido anestesia libre de opioides:

-Hipnosis: Sevorane 1,5\%.

-Relajación muscular: Rocuronio $0,6 \mathrm{mg} / \mathrm{kg}$.

-Analgesia multimodal: Dexmedetomidina $0,5 \mathrm{mcg} / \mathrm{kg} / \mathrm{h}$ (carga $1 \mathrm{mcg} / \mathrm{kg}$ a pasar en 10 minutos), Lidocaína $2 \mathrm{mg} / \mathrm{kg} /$ h peso corregido (carga 1,5 mg/kg a pasar en 10 minutos), Ketamina 0,2 mg/kg + 0,1 mg/kg cada hora, Ketorolac 1 $\mathrm{mg} / \mathrm{kg}$, Paracetamol $10 \mathrm{mg} / \mathrm{kg}$, Dexametasona $0,2 \mathrm{mg} / \mathrm{kg}$ (Max $8 \mathrm{mg}$ ).

•Prevención náuseas y vómitos: Ondasentron 4 mg.

Criterios de inclusión: Pacientes sometidos a cirugía bariátrica en Junio en el hospital universitario austral.

Criterios de exclusión: Pacientes con alergia a cualquiera de las drogas utilizadas.

Pacientes en tratamiento por dolor crónico.

Pacientes que se realicen cirugía bariátrica tipo revisional o de conversión.

Resultados: Se analizó la historia clínica de 10 pacientes de cirugía bariátrica, evaluando los requerimientos de rescates en el post operatorio y la cantidad de días de internación requerida.

El promedio de rescates recibidos fue de: 2,1 rescates de fentanilo $50 \mathrm{mcg}$, siendo 4 rescates el máximo recibido por un paciente y 0 el mínimo.

En cuanto a los días de internación, el promedio fue de 1,3 días de internación. Siendo el máximo 3 días por una complicación correspondiente al área quirúrgico (sangrado post operatorio inmediato).

El $80 \%$ de los pacientes fue dado de alta al día siguiente de la cirugía cumpliendo con los objetivos de trabajo del equipo quirúrgico.

Conclusión: Si bien serían necesarios mayores estudios para corroborar los beneficios los resultados obtenidos en la presente recopilación de casos consideramos que es una técnica válida para realizar, en caso que amerite.

https://doi.org/10.25237/congresoclasa2019.84 